\title{
The pursuit of DLPFC: Non-neuronavigated
}

\section{methods to target the left dorsolateral prefrontal}

cortex with symmetric bicephalic transcranial

\section{Direct Current Stimulation (tDCS)}

\section{Ole Seibt, ${ }^{\text {a,c }}$ Andre R. Brunoni, b Yu Huang,c Marom Bikson,c}

a Department of Medical Technology, Technische Universität Berlin, Berlin, Germany

b Interdisciplinary Center for Applied Neuromodulation, Service of Interdisciplinary

Neuromodulation and Laboratory of Neurosciences (LIM-27), Department and Institute of Psychiatry, University of São Paulo, São Paulo, Brazil

c Department of Biomedical Engineering, The City College of City University of New York, New York, New York

Correspondence: Ole Seibt, Neural Engineering Laboratory, Department of Biomedical Engineering, The City College of New York of CUNY, T-463 Steinman Hall, 160 Convent Avenue, New York, NY 10031, Lab: 212-650-8653, E-Mail: seibt.ole@gmail.com 


\section{Abstract}

Background: The dose of transcranial direct current stimulation (tDCS) is defined by electrode montage and current, while the resulting brain current flow is more complex and varies across individuals. The left dorsolateral prefrontal cortex (IDLPFC) is a common target in neuropsychology and neuropsychiatry applications, with varied approaches used to experimentally position electrodes on subjects. Objective: To predict brain current flow intensity and distribution using conventional symmetrical bicephalic frontal $1 \mathrm{x} 1$ electrode montages to nominally target IDLPFC in forward modeling studies. Methods: Six highresolution Finite Element Method (FEM) models were created from five subjects of varied head size and an MNI standard. Seven electrode positioning methods, nominally targeting IDLPFC, were investigated on each head model: the EEG 10-10 including F3-F4, F5-F6, F7-8, F9-F10, the Beam F3-System, the 5-5cm-Rule and the developed OLE-System were evaluated as electrode positioning methods for 5 x $5 \mathrm{~cm}^{2}$ rectangular sponge-pad electrodes. Results: Each positioning approach resulted in distinct electrode positions on the scalp and variations in brain current flow. Variability was significant, but trends across montages and between subjects were identified. Factors enhancing electric field intensity and relative targeting in IDLPFC include increased inter-electrode distance and proximity to thinner skull structures.

Conclusion: Brain current flow is can be shaped, but not focused, across frontal cortex by tDCS montages, including intensity at IDLPFC. The OLE-system balances IDLPFC targeting and reduced electric field variability, along with clinical ease-of-use.

Keywords: transcranial Direct Current Stimulation (tDCS), Finite Element Method (FEM), left Dorsolateral Prefrontal Cortex (IDLPFC) 


\section{Introduction}

Transcranial direct current stimulation (tDCS) is considered a safe, portable, low cost, easy-to-use neuromodulatory technique that involves non-invasive delivery of weak direct current (typically 1 to $2.5 \mathrm{~mA}$ ) to the brain. The resulting brain electric field (EF), though lowintensity, modulates ongoing brain activity [1], influence synaptic efficacy [2], and produce plastic changes in excitability [3] and behavior [4]. Nearly $20 \%$ of all tDCS studies investigate the neuromodulation of the dorsolateral pre-frontal cortex (DLPFC) [5], including clinical studies indicating promising results for conditions such as schizophrenia, addiction, anxiety disorders and major depression disorder (MDD) [4].

However, despite the use of the left DLPFC (IDLPFC) as a primary or adjunct target in tDCS, the impact of varied electrode placement paradigms (and errors) and anatomical interindividual differences on brain current flow have not been investigated in detail. Notably, stimulation site localization was recognized to be a sensitive issue in repetitive transcranial magnetic stimulation (rTMS) studies where several methods have been proposed, such as the "5-5cm rule", the "6cm rule", the "Beam F3-System", the "EEG 10-10 method" and resonance image guided neuronavigation methods [6][7][8][9]. Conventional bicephalic tDCS produces diffuse current flow between the electrodes, which while less focal than rTMS is still sensitive to changes in electrode montage [10]. Also, in contrast to TMS, in tDCS a fixed current intensity is conventionally used, though, individual anatomical differences may be no less important [11]. The importance and methodology to tanget DLPFC with tDCS (in the context of individual variability) will be fundamentally distinct, but not less important, than in TMS.

These issues are addressed systematically for the first time here, using FEM simulations of current flow.

Individualized stimulation informed by anatomical and functional mapping, is common for neuromodulation approached such as rTMS and Deep Brain Stimulation (DBS). Given the 
unique dosimetry (physics of current delivery to the brain) of tDCS, the role and value of individualized stimulation should be independently considered. Individualized neuromodulation treatment is not without complexity and cost, including scanning (MRI), identification of the target [9], and simplifications in dosimetry (e.g. geometrically projecting a target instead of current flow modeling). Thus there is a gap between clinically practical neuromodulation (e.g. FDA approved TMS) and state-of-the-art research approaches (e.g. neuronavigation). Especially given the cost and deployment advantages of tDCS, here were optimized approaches that would not require neuro-navigation, individualized titration/ segmentation, or functional mapping (TMS, fMRI) and further limited our analysis to conventional 5x5 cm electrodes, as opposed to High-Definition tDCS[4][13][14].

Though our goal was to optimize a low-cost approach, in order to address questions about the role of tDCS electrode position and anatomy, we required high-resolution (gyri-specific) individualized (MRI-derived) forward models [15]. Five adults head sizes, selected to span head sizes, were considered along with theaveraged scan of 152 subjects from the Montreal Neurological Institute (Montreal, Quebec, Canada). Symmetrical frontal bicephalic montages, that do not require functional mapping (e.g. TMS) or neuronavigation (e.g. anatomical MRI), were positioned on each head model. In each case $2 \mathrm{~mA}$ of current was applied through $5 \times 5 \mathrm{~cm}^{2}$ rectangular sponge-pad electrodes. Electrodes were positioned according to: 1) the international EEG 10-10 System including F3-F4, F5-F6, F7-F8 and F9F10; 2) the Beam F3-System; 3) the 5-5cm-Rule and 4) the newly developed "OLE-System" (described below).

Our forward modelingresults illustrate the non-triviality of symmetrical tDCS electrode positioning for IDLPFC modulation including the limits of relying on simplistically placing electrode "over" the target (and borrowing TMS conventions). We develop a novel electrode positioning system (OLE-System), that does not rely on functional imaging, mapping (e.g. 


\section{The pursuit of IDLPFC}

TMS) or neuro-navigation, based on specific criteria such as electric field intensity in lDLPFC andrelative targeting, including accommodating for inter-individual differences.

\section{Methods and Materials}

High-resolution T1-weighted gradient echo 3-T MRI scans were performed on five neurological normal subjects with no history of psychiatric disorders. All images had an isotropic resolution of $1 \mathrm{~mm}^{3}$. Additionally, an MNI-152 head (non-linear $6^{\text {th }}$ generation, [15]), generated by averaging the MRI of 152 subjects at the Montreal Neurological Institute (Montreal, Quebec, Canada), was also used (referenced as MNI standard head in the sequel).

The raw image data was then bias corrected and segmented into six non-overlapping tissue compartments with an automated probabilistic segmentation routine from Statistical Parametric Mapping (SPM8, Welcome Trust Centre for Neuroimaging, London, UK). An inhouse MATLAB script was used to correct for automated segmentation errors [16]. Subsequently, lingering errors in continuity and detail were manually corrected in ScanIP 6.2 (Simpleware Ltd, Exeter, UK) to demarcate boundaries and ensure accuracy and realistic individual anatomy for all subjects (Figure 1). The fat tissue was also manually separated out from the scalp. The final segmented tissue compartments represented gray matter, white matter, cerebral spinal fluid (CSF), air, skull, fat tissue and skin. Additionally, the lDLPFC was manually segmented from gray matter cortex area according to neuroanatomical rules [17] for later visualization of the target area (Figure A.1). Note that the MNI standard head only has a field of view (FOV) down to the nose. To build a whole-head model, another averaged head, developed from 26 subjects by Dr. C. Rorden [16] with a larger FOV, was registered and resliced to the MNI standard head using SPM8. The lower part of the resliced head was then fused with the MNI head to form a whole-head model.

\section{[Figure 1]}


All segmented tissue compartments between the most superior point on C2 (axis) and the most superior point on the scalp (vertex) were measured in order to categorize the scans according to their tissue volume. This was done to compute a range of head sizes for typical adults that may imply subject specific differences in electrode positioning and EF distribution. Seven electrode montages were computed on each of the six heads to account for interindividual differences in brain size and cranial anatomy. The six heads were categorized based on overall head volume and listed from largest to smallest (Table 1) as follows: MNI standard head; Extra Large head (XL): male 36 years old; Large head (L): female 35 years old; Medium head (M): male 40 years old; Small head (S): female 25 years old and an Extra Small head (XS): female 32 years old. The use of different head volumes is crucial since recent studies indicated subject specific variability in susceptibility to the same dose [11].

\section{[Table 1]}

Eight equally sized CAD-Models of $5 \times 5 \mathrm{~cm}^{2}$ sponge-pad electrodes were created in SolidWorks 2013 (Dessault Systems Corp., Waltham, MA) and imported as Standard Tessellation Language (STL) files to ScanCAD (Simpleware Ltd, Exeter, UK) for positioning on each respective head model. The active (red) sponge-pad electrode assemblies were placed over the left frontal lobe of each subject and the return electrode (blue) over the contralateral position representing (Figure 2).

\section{[Figure 2]}

We tested four different positioning methods over DLPFC. The first three are already applied in clinical practice while the fourth was tested for the first time in the present study. All approaches aim to ensure feasibility and anatomical targeting reproducibility:

1) EEG 10-10 including "F3-F4": the international EEG 10-10 system [8] through universal electrode caps for standardized head sizes. This is a widely used approach, at least for non-neuronavigated methods. Predefined coordinates that resembled virtually fitted F3- 
F4 positions that were generated in an earlier study [16] were used to centrally align the sponge-electrodes for montages A. However, in clinical practice numerous measurements and calculations that can be excessively time consuming would be necessary to localize the respective coordinates for each individual head. Therefore, alternative options to the EEG system are commonly employed. Three additional $5 \times 5 \mathrm{~cm}^{2}$ sponge-pad electrode montages were imported and centrally positioned on EEG 10-10 scalp locations over F5-F6, F7-F8 and F9-F10 with the anode over the left and the cathode over the right hemisphere. These lateralized bicephalic electrode montages were imported for later data presentation and trend line extrapolation.

2) The "Beam F3-System" [7] that uses nasion to inion, tragus to tragus distances and head circumference to calculate electrode center coordinates on the scalp for each subject using the "Beam F3 Shortcut Software" (www.clinicalresearcher.org/software.htm). Two additional sponge-pad electrode assemblies were imported and centrally positioned on the resulting coordinates calculated by the software to constitute montage B that is clinically applied through the use of the EasyStrap (www.soterixmedical.com/tdcs/ accessories).

3) The "5-5-cm rule", derived from rTMS-based positioning, is a straightforward and usually employed positioning method for tDCS over DLPFC. In this, the sponge-pad electrodes are positioned 5cm anterior to the motor cortex (M1) along the parasagittal line [6]. Here, the M1 location was approximated 5cm laterally from the vertex. The electrodes were then positioned $5 \mathrm{~cm}$ anterior to the approximated $\mathrm{M} 1$ location with the dorsal sponge edge parallel aligned to the eyebrows as depicted in montages D.

4) Additionally, a fourth reproducible positioning method, deploying a modified EasyStrap engineered in three sizes (Large, Medium, Small), was derived to minimize the time cost for pre-treatment measurements without compromising lDLPFC targeting accuracy and EF focality. The "Omni-Lateral-Electrode-System" (OLE-System) uses a centrally 


\section{The pursuit of IDLPFC}

engraved point marker on the occipital strap to accurately place the center position on inion. In clinical practice, the position is secured and held in place through a chin strap with the strap hinge over T7/T8. In this, the most superior point on the pinna may be used as a second anatomical landmark to place the hinge for alignment. A standardized angle adjustment of $165^{\circ}$ between occipital and electrode strap was modeled for montage $\mathrm{C}$ on each head with a dorsal electrode edge distance of $10 \mathrm{~cm}$ (Figure 3).

[Figure 3]

The FEM model generation required a three-dimensional volume mesh generation of the imported electrode montages and the seven segmented tissue compartments. An adaptive (+FE-Free, ScanIP) mesh algorithm was used to reduce the number of elements and the degrees of freedom without compromising the computational accuracy in order to produce a compact mesh size. A compound coarseness of - 17 was applied. The resolved volume entailed approximately $9.5 \times 10^{6}$ tetrahedral elements with about $13.0 \times 10^{6}$ degrees of freedom leading to a highly detailed mesh that requires no further smoothing of tissue layers after segmentation errors were corrected. This is essential for the accuracy of the FEM simulation since smoothing operations may produce discontinuities in anatomic specific tissue compartment architecture.

A total of 42 FEM models (seven electrode montages positioned on six heads) were then created in Comsol Multiphysics (COMSOL, Inc., Burlington, MA). Electrostatic volume conductor physics were applied. All exterior boundaries were treated as electrically insulated and all interior boundaries were treated as continuous across interfaces. The exterior boundaries of the anode were then used to apply $2 \mathrm{~mA}$ of normal inward current flow leading to an injected current density of approximately $0.8 \mathrm{~A} / \mathrm{m}^{2}$. Constant inward current flow was retained for all models in order to produce comparable results. The cathode was set to ground $(\mathrm{V}=0)$ on its exterior boundaries. Material properties were assigned to the generated masks in 
the respective sub domain setting. Isotropic electrical conductivity values (in $\mathrm{S} / \mathrm{m}$ ) have been assigned as follows: air,1x10-15; skin, 0.465; fat, 0.025; skull, 0.01; CSF, 1.65; gray matter, 0.276; white matter, 0.126; electrodes, $5.99 \times 10^{7}$ and saline-soaked sponge pads, 1.4 [18]. A linear solver with conjugate gradients and algebraic multigrid preconditions was applied with a relative tolerance of $1 \mathrm{e}-6$ in order to solve the Laplace-Equation $(\nabla \cdot(\sigma \nabla V)=0$.

\section{Results}

We predicted the resulting brain current flow during symmetrical bicephalic tDCS with a nominal target of IDLPFC. Though a total of seven montages by six heads were simulated in this study, for detailed illustration, we consider four heads (MNI, extra large, medium, small; Figure 4,5,6,7) and four positioning guidelines (F3-F4 (panels A), the Beam F3-System (panels B), the OLE-System (panels C) and the 5-5cm-Rule (panels D); Figure 4,5,6,7).

Volumetric EF and current density (CD) plots of gray- and white-matter were generated to compare current flow distribution depending on individual head anatomy and electrode positioning method. Same-scale plots were generated for direct comparison: EF magnitudes above $0.8 \mathrm{~V} / \mathrm{m}$ were treated as $0.8 \mathrm{~V} / \mathrm{m}$ and CD magnitudes exceeding $0.2 \mathrm{~A} / \mathrm{m}^{2}$ were treated as $0.2 \mathrm{~A} / \mathrm{m}^{2}$ for data illustration. Absolute peak values, for the region shown, were depicted adjacent to each plot. The IDLPFC was separately segmented as target area and included as

volumetric EF plot (Figure 4,5,6,7 in panel A.1, B.1, C.1 and D.1) to evaluate stimulation focality and targeting.

Across simulated conditions, brain current flow was broadly distributed with peak EF intensities generally induced in the intermediate frontal lobe areas between the electrodes. Absolute EF magnitudes ranged from $0.66 \mathrm{~V} / \mathrm{m}$ (MNI head with 5-5cm-Rule montage) to $2.44 \mathrm{~V} / \mathrm{m}$ (XS head with F7-F8 montage), representing a $\sim 4$ fold increase in EF magnitude. A comparable relative variability was predicted for IDLPFC with peak EF intensities ranging 
from $0.30 \mathrm{~V} / \mathrm{m}$ (MNI head with 5-5cm-Rule montage) to $1.16 \mathrm{~V} / \mathrm{m}$ (XS head with F5-F6 montage).

These EF fluctuations were induced by: 1) A few cm in displacement in electrode position; and 2) $\mathrm{A} \sim 30 \%$ decrease in inter-individual head volume. Assuming such changes in scale influence outcomes of tDCS, the role of electrode positioning system across individual differences is significant.

[Figure 4]

[Figure 5]

[Figure 6]

[Figure 7]

\section{Electrode Position across Individuals - Brain Current Flow Intensity}

The EF maxima for each electrode positioning system are illustrated as box plots for IDLPFC (black) and overall cortical values (red) across six heads, including MNI (Figure 8). Generally, peak EF values augmented for the OLE-System, the F5-F6 and F7-F8 electrode montages. The ratio of IDLPFC peak EF to the overall cortical peak EF is one measure for relative stimulation targeting. Averaged across subjects (Figure 8, green circle), this targeting ratio ranged from a minimum of 0.35 (F9-F10) to a maximum 0.60 (OLE-System). This indicated improved relative targeting with the OLE-System.

An increase in standard deviation indicates an increase in EF peak variability across subjects for the same positioning system. The cortical EF variability, across subjects, ranged from $\pm 0.38 \mathrm{~V} / \mathrm{m}$, thus $45 \%$ of mean peak value (for the Beam F3-System) to $\pm 0.63 \mathrm{~V} / \mathrm{m}, 50 \%$ of mean peak EF (for F9-F10). The nominally targeted IDLPFC was exposed to relatively 
smaller EF variability ranging from $\pm 0.09 \mathrm{~V} / \mathrm{m}, 16 \%$ of the mean IDLPFC peak EF (for F9F10) to $\pm 0.23 \mathrm{~V} / \mathrm{m}, 27 \%$ of mean IDLPFC peak EF (for F5-F6).

Given that intra-subject variability could exceed sensitivity to electrode positioning methods, we turned our attention to inter-individual differences.

[Figure 8]

\section{Inter-Individual Differences in Head Volume - Brain Current Flow Intensity}

The MNI standard head was used to normalize (predict) response across heads for each positioning methods. Overall cortical and IDLPFC peak EF values were plotted over a normalized (to the MNI standard head) cranial tissue volume (Figure 9). Note that each specific head in this study was smaller in total head volume than the MNI standard head

(Table 1). Across montages, the MNI standard head with the largest tissue volume produced the lowest EF intensities. The XS head with the smallest tissue volume produced the highest EF intensities. An average (across montages) 2.4 fold increase in peak EF (with a standard deviation of 0.23 ) was observed from the XS to the MNI for all electrode positions. This corresponds to a $\sim 30 \%$ decreases in brain (and cranial) tissue volume. For intermediate head-sizes, there was a trend (but noisy across montages) toward increasing peak EF magnitudes with a decrease in head tissue volume; in no case where overall peak EF lower than the MNI head. Therefore, EF intensity fluctuations generally correlated with gross anatomical differences, such as head tissue volume.

[Figure 9]

\section{Electrode Position across Individuals - Brain Current Flow Distribution}

Electrode montages with an anterior and inferior electrode position (relative to F3-F4) resulted in an increased EF in the frontal lobe and, more specifically, in IDLPFC (e.g. with the 
OLE-System, F5-F6 or F7-F8). Posterior displacement led to an increasingly diffuse EF spread over the partial-lobe away from IDLPFC (e.g. with the Beam F3-System and 5-5cm Rule). In comparison, electrode placement according to OLE System lead to an EF that was 〜1.4 (F3-F4) to 2.0 fold larger (5-5cm-Rule or Beam F3-System) in lDLPFC.

Bidirectional radial EF plots (Figure 4,5,6,7 in panel A.2, B.2, C.2 and D.2) were generated in section views around IDLPFC (wireframe) to evaluate the distribution of cortical inward (red), and outward current flow (blue) across multiple gyri and sulci near the target. Radial EF increased with the overall EF intensities and, consequently, correlated with head tissue volume (Figure 4,5,6,7 in panel A2, B2, C2 and D2). Current flow through the lDLPFC surface boundary with CSF was always (throughout all computed heads and montages) predominantly inward under the anode (Table 2). Idiosyncratic cortical folding of lDLPFC caused $15.1 \%$ deviation around an average rate of $67.3 \%$ anodal (inward) current flow across all heads. Note that the exclusion of the MNI standard head reduced the deviation to $4.8 \%$ around an average rate of $60.9 \%$ anodal current flow.

\section{[Table 2]}

CD intensities were also plotted in coronal sections with a vector field (cones) to illustrate current flow patterns relative to the cortical surface. Each coronal section was selected for the coordinate of cortical peak CD intensity in the frontal lobe (Figure 4,5,6,7 in panel A.3, B.3, C.3 and D.3). An anterior coronal slice coordinate shift, towards IDLPFC, was observed with the F3-F4 and the OLE-System. Additional, CD peaks were observed at gray- and white matter intersections with CSF ventricles. Notably, a local CD maximum was predicted in all 42 simulations, at the intersection of fornix and corpus callosum with the third and lateral ventricles and in anterior white-matter regions that encompass the lateral ventricles (not shown).

\section{Geometric and Anatomical Factors Governing Current Flow}




\section{The pursuit of IDLPFC}

We considered the geometric factors effecting brain current flow by systematically measuring: 1) Angle between the electrode center and inion from the most dorsal point on the ear (in the medial sagittal plane); and 2) Electrode distance across the scalp (Figure 3). The peak EF in IDLPFC was plotted over electrode angle and distance for F3-F4 (panels A), the Beam F3-System (panels B), the OLE-System (panels C) and the 5-5cm-Rule (panels D) on each head (Figure 4,5,6,7), extrapolated with additional results from F5-F6, F7-F8 and F9F10 (EEG 10-10) scalp locations and fitted with a three-parameter exponential function. A rise in peak EF intensities was observed for electrode positioning angles between 140 and $165^{\circ}$ and dorsal electrode edge distances (across the scalp) from 4 to $10 \mathrm{~cm}$ (Figure 10) due to: 1) The facilitation of current injection through thin skull structures around the temporal bone; and 2) Decreased scalp shunting.

The OLE-System (Figure 3) was subsequently derived, for improved performance as an ad hoc positioning system, with an electrode angle of $165^{\circ}$ between the occipital- and the electrode strap (EasyStrap) and an electrode distance of $10 \mathrm{~cm}$ across the scalp (measured along the electrode strap). The midpoint of occipital strap was centered over inion $\left(\mathrm{I}_{\mathrm{z}}\right)$ and the hinges that link occipital-, electrode- and chin strap were placed over the most dorsal point on the ear for positioning.

[Figure 10] 


\section{Discussion}

The IDLPFC is a common target in neuromodulation for therapy and cognitive enhancement, based on its role in attention and mood networks. While neither its unique role in brain function nor the targeting ability of any neuromodulation intervention should be exaggerated, to optimize neuromodulation to maximally influence IDLPFC is a rational approach. This computational FEM modeling study is the first to systematically optimize tDCS for IDLPFC modulation. Our goal was to determine a tDCS sponge-pad electrode montage for maximal EF delivery to IDLPFC assuming that a higher EF magnitude increases the degree of neuromodulation under the quasi-uniform assumption [19]. We restricted ourselves to symmetrical bicephalic sponge-pad-electrodes (as opposed to asymmetrical or High-Definition tDCS [12][13][14]) as our focus was to approximate the most common and simple method for clinical application. Our goal was to develop a single montage that is optimized across subjects, and so we modeled different head sizes ("Extra Small", "Small", "Medium", "Large", "Extra Large", and the "MNI" standard head). Thus our final recommendation is for the "OLE-System" (montage) which is simple to apply and reproducible across subjects.

Several basic findings reinforced predictions from previous modeling efforts including: 1) Conventional tDCS montages produce diffuse current flow between electrodes [14][20]; 2) with clustering of "hot-spots" and peak current density not necessarily under an electrode [14][20][21][22]; 3) application of the same dose (electrode montage and current intensity) across subjects produces varied brain current flow patterns and intensity [11]. Nevertheless, our detailed analysis of montage variations indicates current flow patterns can be controlled, and, within limitations, optimized by electrode positioning system.

A fundamental challenge in non-invasive dose optimization is a poor correspondence between landmarks measurable on the scalp and underlying brain targets. The universally 
used EEG 10-10 scalp coordinate system is arbitrary and, as exhaustively studied for TMS, other scalp positioning systems, such as “5-5cm-Rule" and "Beam F3-System" suggest different coil positions. With tDCS, and more generally tES, the further challenge is that current flow is not restricted, or necessarily maximal, under the electrode position - so even if the scalp position directly over IDLPFC is known, this does not simply correspond to the optimal electrode position to stimulate IDLPFC. We therefore considered various existing (various EEG 10-10, 5-5cm-Rule, Beam F3-System) and customized (OLE-System) scalp electrode positioning systems based on the criterion of maximal current delivery to IDLPFC.

The electrode positions following EEG 10-10 system were fully generated in the virtual environment [16]. The software provides the exact 3D coordinates of the electrodes in the original MRI coordinate system, which can facilitate actual live electrode placement and proper cap adjustment to the individual head (see [20] for example). If possible, one can use commercially available electrode positioning systems to guide this adjustment (e.g. Polhemus tracker). We have not done so in this work as it only focuses on modeling.

Based on the assumptions and goals stated above, we found the OLE-System optimal. This result was not unanticipated since we designed the new OLE based on our goals and through understanding of the underlying biophysics of current flow based on head anatomy. Specifically the OLE system balances: 1) increased current flow as electrode distance is increased (due to decreases scalp shunting); 2) peak current flow when electrodes span across rather than over a target; 3) role of variation in bone thickness (thus positioning in close proximity to the temporal bone). EEG 10-10, Beam F3-System and the 5-5cm-Rule lead to maximum current flow in the superior- and middle frontal gyrus, depending on the cortex anatomy. The predicted average IDLPFC peak intensities were 15\% higher with OLE compared to F3-F4, 45\% elevated in comparison to 5-5cm-Rule and 55\% increased compared to Beam F3 System. Peak EF intensity also increased with a decrease in overall head tissue volume. The highest EF intensity, in five out of six heads, was achieved with an 
F5-F6 montage. However, the overall cortical peak EF intensities increased disproportionally over IDLPFC. This indicates, accompanied by earlier results [20], deteriorating stimulation focality with an increase in stimulation intensity. The ratio of peak EF in IDLPFC to overall peak EF was optimal with the OLE-System for three- (including the MNI standard head), and with the F3-F4 for two heads.

Interestingly, despite significant differences in gross anatomy and a high degree in cortical folding, in all cases simulated with an anode over the left hemisphere, radial current crossing from CSF into IDLPFC was dominantly inward (excitatory). Though, there was significant variation in the intensity of EF in IDLPFC across subjects and montages, the relative ratio of inward to outward current was relatively constant $(<5 \%$ variation across subjects/montages, excluding MNI).

Because individualized modeling is expensive in imaging and computational resources, we wanted to propose a general system across subjects (not requiring MRI, neuro-navigation, TMS mapping, etc.). In addition, the OLE system is practically more robust to apply and easier to be learned and used by technicians and patients (for instance, in high-throughput and home-treatment settings) because the OLE system uses a coordinate system built into the head-positioning strap itself - no additional measurements (EEG 10-10) are needed. The OLE strap is positioned on the subject's head using natural landmarks and grid-lines that identify where to insert electrodes.

Note, that considerable variability in the exact cortical location and extension of DLPFC was reported [23], thus, a cortical target may not simply be identified by cortical landmarks such as gyri 0r sulci boundaries. Therefore, functional differences and clinical significance of these predictions remain to be assessed. However, if one assumed the efficacy of prior tDCS trials were indeed promoted by current delivery to IDLPFC, then future trials should caution that positioning electrodes according to F3-F4 or methods adapted from TMS are not 
optimized compared the OLE system. In regards to reluctance to change from a historical (non-optimal) montage simply because the historical positioning-system "worked", we note the OLE-system: 1) does not produce brain current flow qualitatively different than F3-F4; 2) reduces between subject current flow variability (and hence potentially outcome variability); 3) increases maximal peak current to other brain regions (pad-based tDCS is inherently not focal and other brain regions may also be relevant targets); and 4) as discussed above, is more simple and robust to position.

Quantitatively, the accuracy of FEM models is limited owing to the lack of representation of cortical architecture smaller than 1 mmand anisotropic electrical properties of cranial tissue. Forward modeling studies may be optimized through conductivity mapping via Diffusion Tensor Imaging (DTI) to incorporate varying fiber orientation and tissue heterogeneity. However, individual cortical folds, CSF layer thickness and ventricle size as well as skin, fat layer thickness and skull architecture were captured accurately to represent inter-individual differences. We draw conservative conclusions to support our recommendation, not relying on results in any given head but rather projecting generalities observed across all heads that can be reasonably expected to extend to wider population.

In conclusion, based on constraints and goals for modulation intensity, and moreover for reproducibility and ease-of-use, we recommend the application of the OLE-System for future tDCS studies targeting the IDLPFC.

\section{Acknowledgements}

ARB is supported by the following grants: 2013 NARSAD Young Investigator from the Brain \& Behavior Research Foundation (Grant Number 20493), 2013 FAPESP Young Researcher from the São Paulo State Foundation (Grant Number 20911-5) and National Council for Scientific and Technological Development (CNPq, Grant Number 470904). MB is supported by grants from NIH (NCI, NINDS), NSF, Wallace H Coulter Foundation, Epilepsy 
Foundation, and the DoD (AFOSR). The City University of New York has patents on brain stimulation with Marom Bikson as inventor. MB has equity in Soterix Medical Inc.

\section{Appendices}

[Figure A.1] 


\section{References}

[1] Reato D, Rahman A, Bikson M, Parra LC, "Low-intensity electrical stimulation affects network dynamics by modulating population rate and spike timing." J Neurosci., vol. 30, no. 45, 15067-79, 2010.

[2] Bikson M, Inoue M, Akiyama H, Deans J K, Fox J E, Miyakawa H, J efferys J G, "Effects of uniform extracellular DC electric fields on excitability in rat hippocampal slices in vitro." J Physiol, vol. 557, no.1, 175-190, 2004.

[3] Nitsche MA, Paulus W, "Excitability changes induced in the human motor cortex by weak transcranial direct current stimulation." J Physiol., vol. 527, no. 3, 633-639, 2000.

[4] Brunoni AR, Nitsche MA, Bolognini N, Bikson M, et al., "Clinical research with transcranial direct current stimulation (tDCS): Challenges and future directions." Brain Stimulation, vol. 5, no. 3, 175-195, 2011.

[5] Kuo MF, Paulus W, Nitsche MA, "Boosting Focally-Induced Brain Plasticity by Dopamine." Cerebral Cortex, vol. 18, no. 3, 18648-651, 2010

[6] Rusjan PM, Barr MS, Farzan F, et. al., "Optimal transcranial magnetic stimulation coil placement for targeting the dorsolateral prefrontal cortex using novel magnetic resonance image-guided neuronavigation." Human Brain Mapping, vol. 31, no. 11, 1643-1652, 2010

[7] Beam W, Borckhardt J, Reeves S, George M, "An efficient and accurete new method for locating the F3 position from prefrontal TMS applications.” Brain Stimulation, vol. $2,2009$.

[8] Klem GH, Lueders HO, Jasper HH, Elger C, "The ten-twenty electrode system of the International Federation. Electroencephalogramm." Clinical Neurophysiology, vol. 52, 1999.

[9] Mylius V, Ayache SS, Ahdab R, et. al., "Definition of DLPFC and M1 according to anatomical landmarks for navigated brain stimulation: Inter-rater reliability, accuracy, 
and influence of gender and age." Neuroimage, vol. 78, 224-232, 2013

[10] Nitsche MA, Doemkes S, Karaköse, Antal A, Liebetanz D, Lang N, Tergau F, Paulus W, "Shaping the Effects of Transcranial Direct Current Stimulation of the Human Motor Cortex.", J ournal of Neurophysiology, vol. 97, no. 4, 3109-3117, 2007.

[11] Datta A, Truong DQ, Mihnas P, Parra L, Bikson M, "Inter-individual variation during transcranial direct current stimulation and normalization of dose using MRI-derived computational models." Frontiers in Psychiatry, vol.3, 2012.

[12] Edwards D,Cortes M, Datta A, Parra L, Minhas P, "Physiological and modeling evidence for focal transcranial electrical brain stimulation in humans: A basis for highdefinition tDCS ." Neuroimage, vol.74, 266-275, 2013.

[13] Villamar MF, Wivatvongvana P, Patumanond J, Bikson M, Truong D, Datta A, Fregni F, "Focal Modulation of the Primary Motor Cortex in Fibromyalgia Using $4 \times 1$-Ring High-Definition Transcranial Direct Current Stimulation (HD-tDCS): Immediate and Delayed Analgesic Effects of Cathodal and Anodal Stimulation." The J ournal of Pain, vol. 14, no. 4, 371-383, 2013.

[14] Datta A, Bansal V, Diaz J, Patel J, Reato D, Bikson M, "Gyri-precise head model of transcranial direct current stimulation; improved spatial focality using a ring electrode versus conventional rectangular pad." Brain Stimulation, vol. 2, no. 4, 201-207, 2009.

[15] Grabner G, Janke AL, Budge MM, Smith D, Pruessner J, Collins DL, "Symmetric atlasing and model based segmentation: an application to the hippocampus in older adults", Medical Image Computing and Computer Assisted Intervention, MICCAI, vol. 9, 58-66, 2006.

[16] Hunag Y, Domchowski JP, Su Y, et al., "Automated MRI segementation for individualized modeling of current flow in the human head." Journal of Neural Engineering, vol. 10, no.6, 2013.

[17] Al-Hakim R, Fallon J, Nain D et al., "A Dorsolateral Prefrontal Cortex Semi-Automatic Segmenter.” Medical Imaging 2006: Image Processing, vol. 10, no. 1, 2006.

[18] Wagner T, Fregni F, Fecteau S, Grodzinisky A, Zahn M, Pascual-Leone A, "Transcranial direct current stimulation: A computer-based human model study." 
Neuroimage, vol. 35, no. 3, 1113-1124, 2007.

[19] Bikson M, Dmochowski J , Rahman A "The "quasi-uniform" assumption in animal and computational models of non-invasive electrical stimulation." Brain Stimulation, vol. 6, no. 4, 704-705, 2013.

[20] Dmochowski J P, Datta A, Bikson M, Su Y, Parra LC, "Optimized multi-electrode stimulation increases focality and intensity at target." J ournal of Neural Enginerring, vol. 8 no. $4,2011$.

[21] Peterchev AV, Wagner TA, Miranda PC, Nitsche MA, Paulus W, Lisanby SH, PascualLeone A, Bikson M, "Fundamentals of transcranial electric and magnatic stimulation dose: definition, selection, and reporting practices." Brain Stimulation, vol. 5, no. 4, 435-453, 2012.

[22] Dmochowski J P, Datta A, HuangY, Richardson J D, Bikson M, Fridriksson J , Parra LC, "Targeted transcranial direct current stimulation for rehabilitation after stroke." Neuroimage, vol 75, no. 1, 12- 19, 2013.

[23] Cieslik EC, Zilles K, Caspers S, Roski C, Kellermann TS, J akobs O, Langner R, Laird AR, Fox PT, Eickhoff SB, "Is There "One” DLPFC in Cognitive Action Control? Evidence for Heterogeneity From Co-Activation-Based Parcellation.” Cerebral Cortex, vol. 256, 2013. 


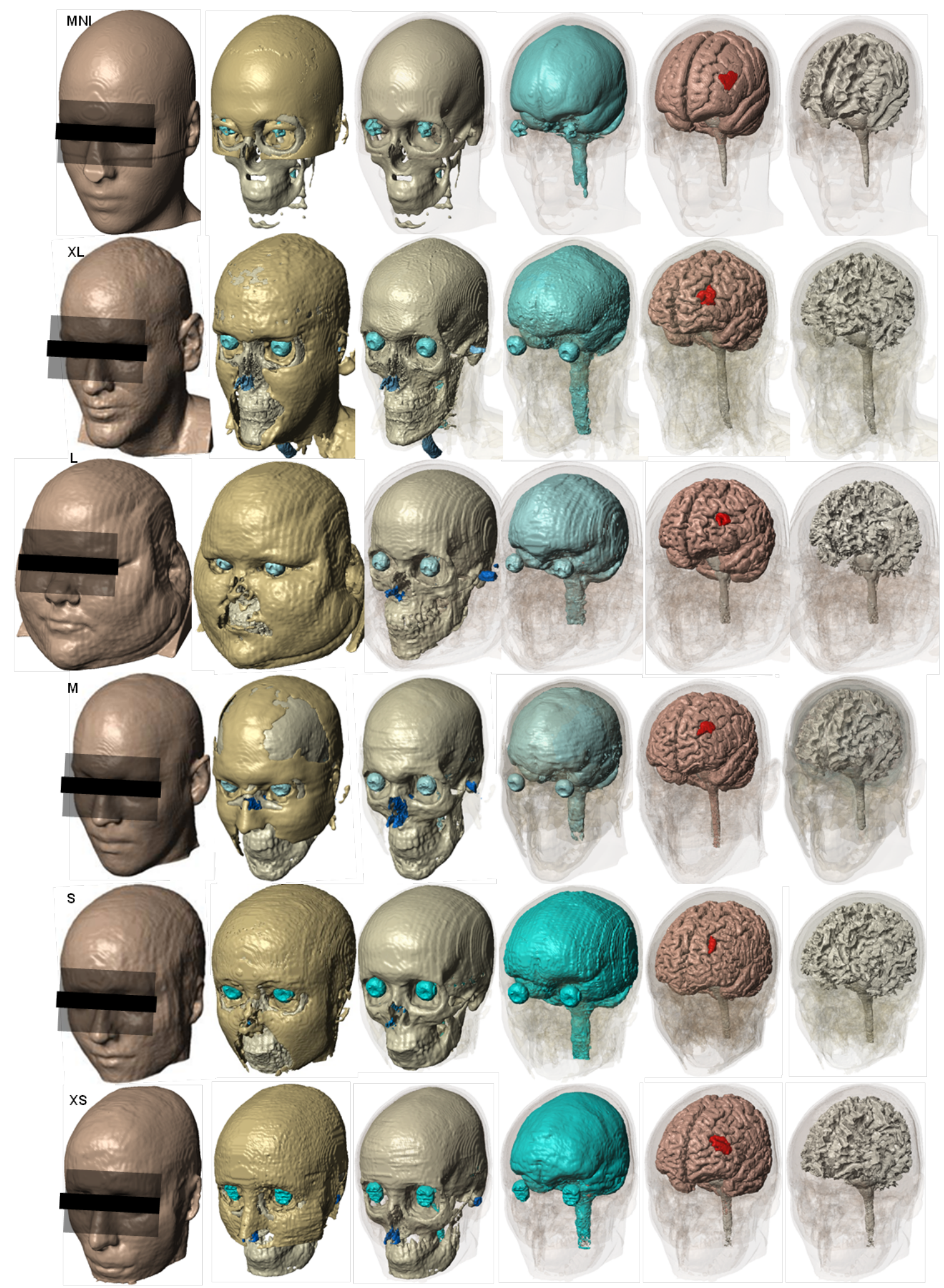

Figure 1: Segmented tissue compartments representing skin, fat, skull, cerebral spinal fluid, gray- and white matter (left to right) of an MNI standard (MNI), extra large (XL), large (L), medium (M), small (S) and an extra small head (XS), categorized based on overall head volume. The IDLPFC (red) was additionally segmented for later evaluation of anatomical targeting. Also, all models include air (dark blue) in the upper respiratory tract and the auditory channels. 


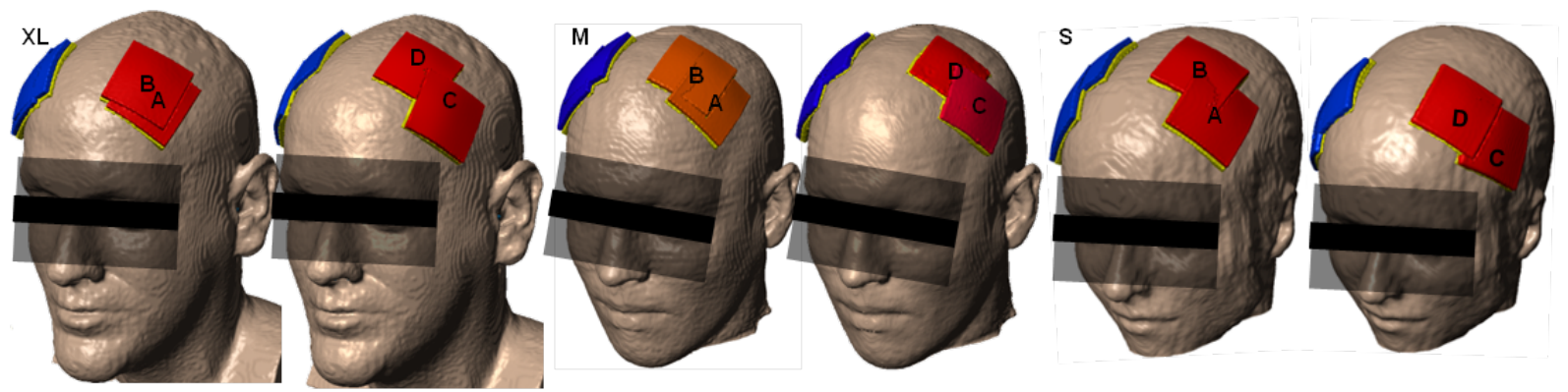

Figure 2: Deployed electrode positioning methods for IDLPFC targeting on three exemplary heads. Bilateral F3-F4 electrode montage positioning according to F3-F4 (A), Beam F3-System (B), OLESystem (C) and the 5-5cm-Rule (D) on a large (L), medium (M) and small sized head (S). 


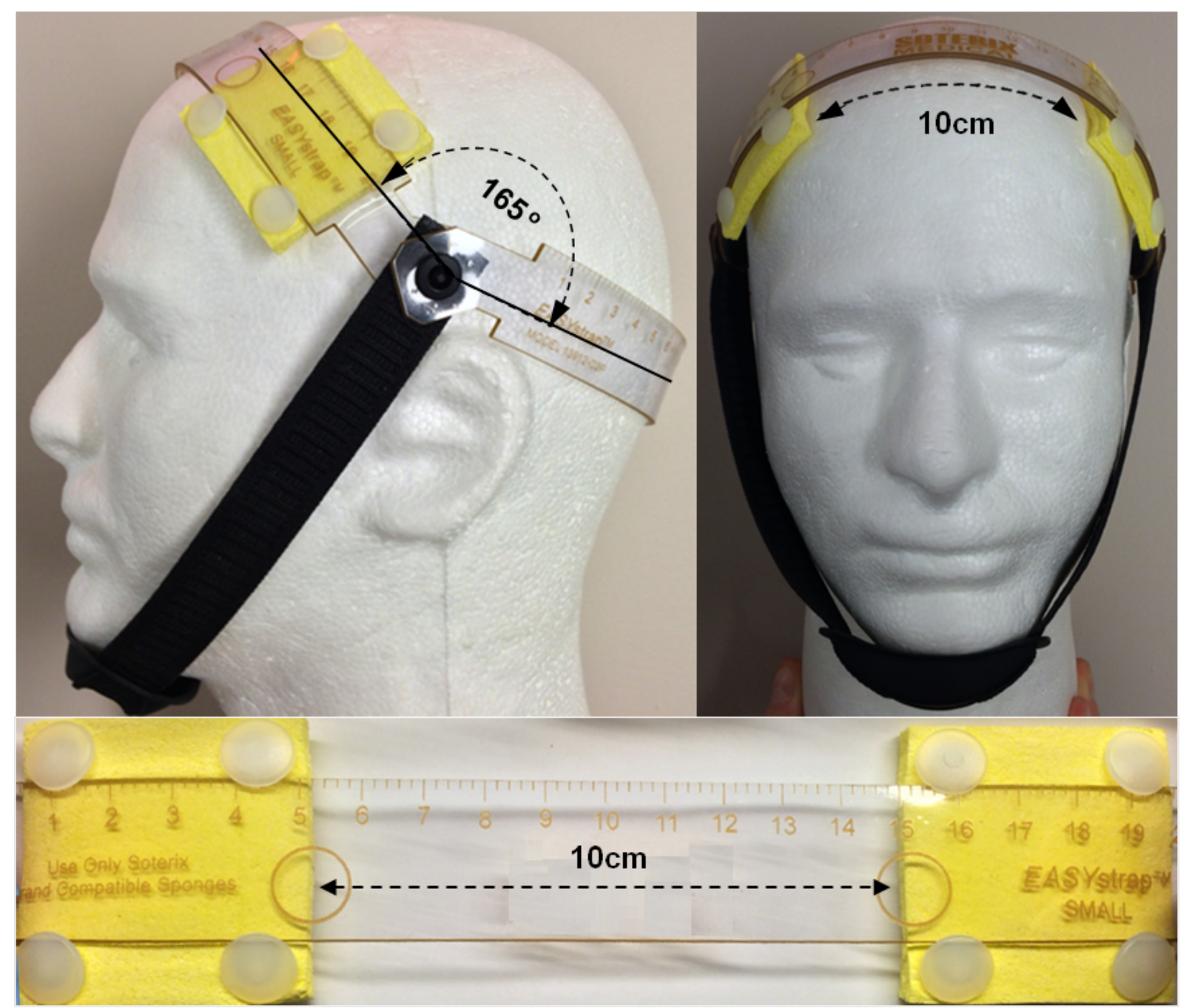

Figure 3: Omni-Lateral-Electrode System placement procedure. The OLE-System placement procedure is deployed as follows: 1) Select EasyStrap size (small, medium, large); 2) Place the midpoint of occipital strap over inion $\left(\mathrm{I}_{z}\right)$; 3) Position the hinges that link occipital-, electrode- and chin strap over the most dorsal point on the ear; 4) Adjust the angle between occipital and electrode strap to $165^{\circ}$ and the distance across the scalp between the dorsal electrode edges to $10 \mathrm{~cm}$. 


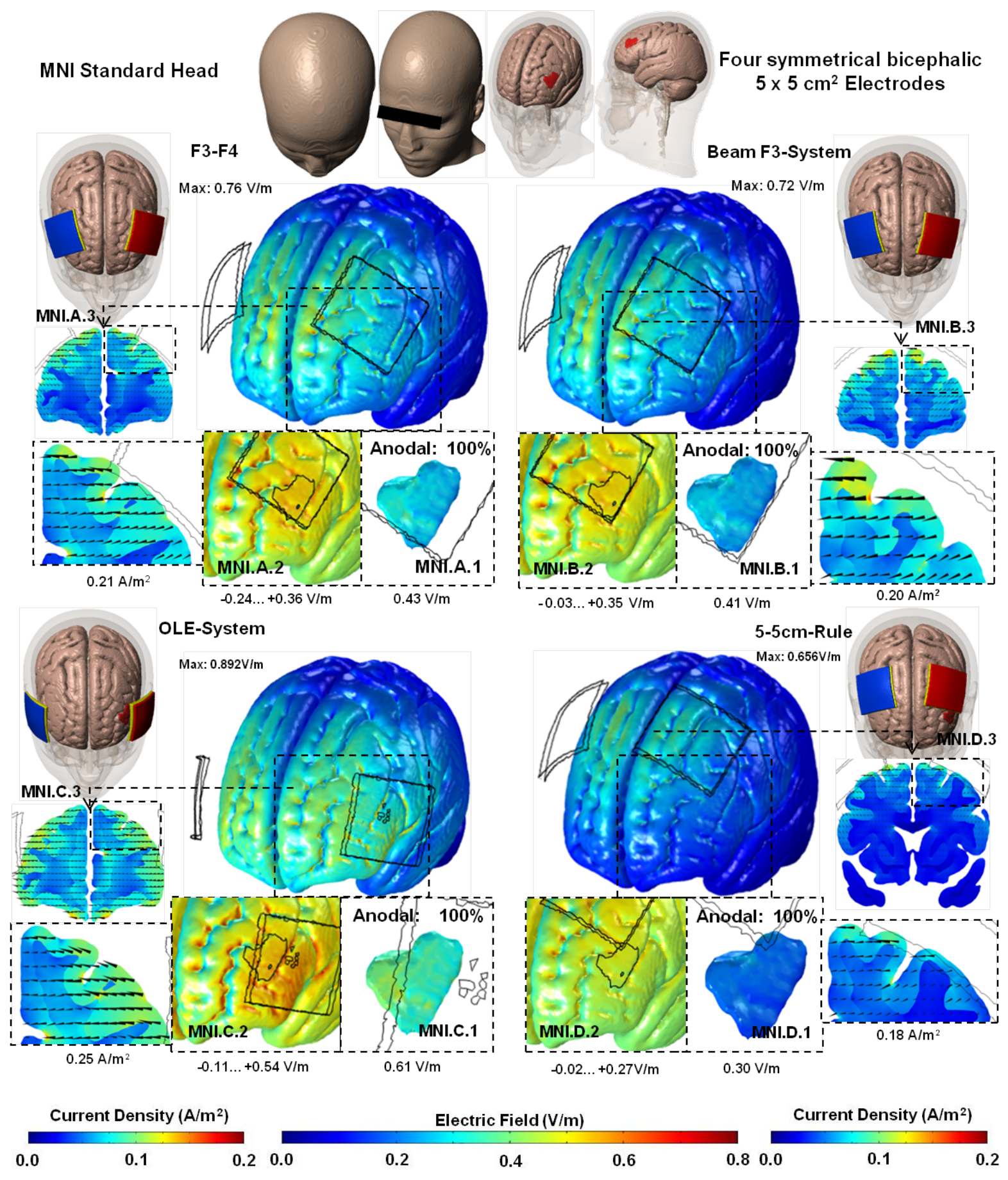

Figure 4: Comparison of brain current flow produced by four localization methods on an MNI standard head. Four methods are compared: "F3-F4" positioning according to the EEG 10-10 (MNI.A), "Beam F3-System" (MNI.B), "OLE-System" (MNI.C) and the "5-5cm-Rule" (MNI.D). 2mA, injected from the anode (red) to cathode (blue), resulted in the depicted electric field across the cortical surface. Crosssection views of the brain, selected for maximal intensity (MNI.A.3, MNI.B.3, MNI.C.3 and MNI.D.3), with magnified insets also showing the resulting vector field (cones), depict the produced current density distribution. The radial electric field across the lateral prefrontal region is shown for each case (MNI.A.2, MNI.B.2, MNI.C.2 and MNI.D.2); scaled to $\pm 0.8 \mathrm{~V} / \mathrm{m}$. The cortical electric field distribution in DLPFC (wireframe) is shown separately with the rate of anodal (inward) current flow (MNI.A.1, MNI.B.1, MNI.C.1 and MNI.D.1); scaled to $0.8 \mathrm{~V} / \mathrm{m}$. Resulting peak intensities are depicted adjacent to each plot. 


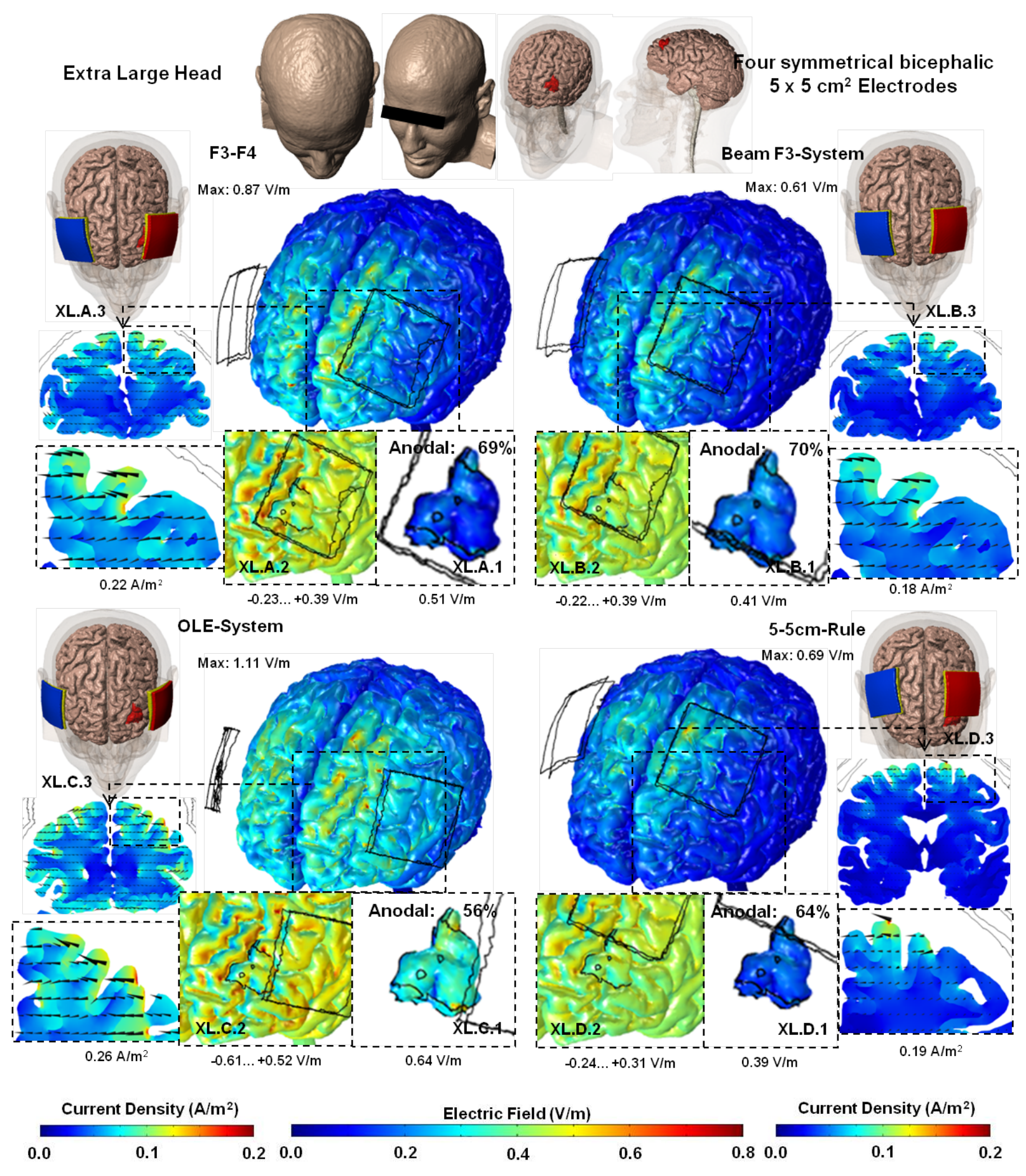

Figure 5: Comparison of brain current flow produced by four localization methods on an extra large head size. Four methods are compared: "F3-F4" positioning according to the EEG 10-10 (XL.A), "Beam F3-System" (XL.B), "OLE-System" (XL.C) and the "5-5cm-Rule" (XL.D). 2mA, injected from the anode (red) to cathode (blue), resulted in the depicted electric field across the cortical surface. Cross-section views of the brain, selected for maximal intensity (XL.A.3, XL.B.3, XL.C.3 and XL.D.3), with magnified insets also showing the resulting vector field (cones), depict the produced current density distribution. The radial electric field across the lateral prefrontal region is shown for each case (XL.A.2, XL.B.2, XL.C.2 and XL.D.2); scaled to $\pm 0.8 \mathrm{~V} / \mathrm{m}$. The cortical electric field distribution in DLPFC (wireframe) is shown separately with the rate of anodal (inward) current flow (XL.A.1, XL.B.1, XL.C.1 and XL.D.1); scaled to $0.8 \mathrm{~V} / \mathrm{m}$. Resulting peak intensities are depicted adjacent to each plot. 


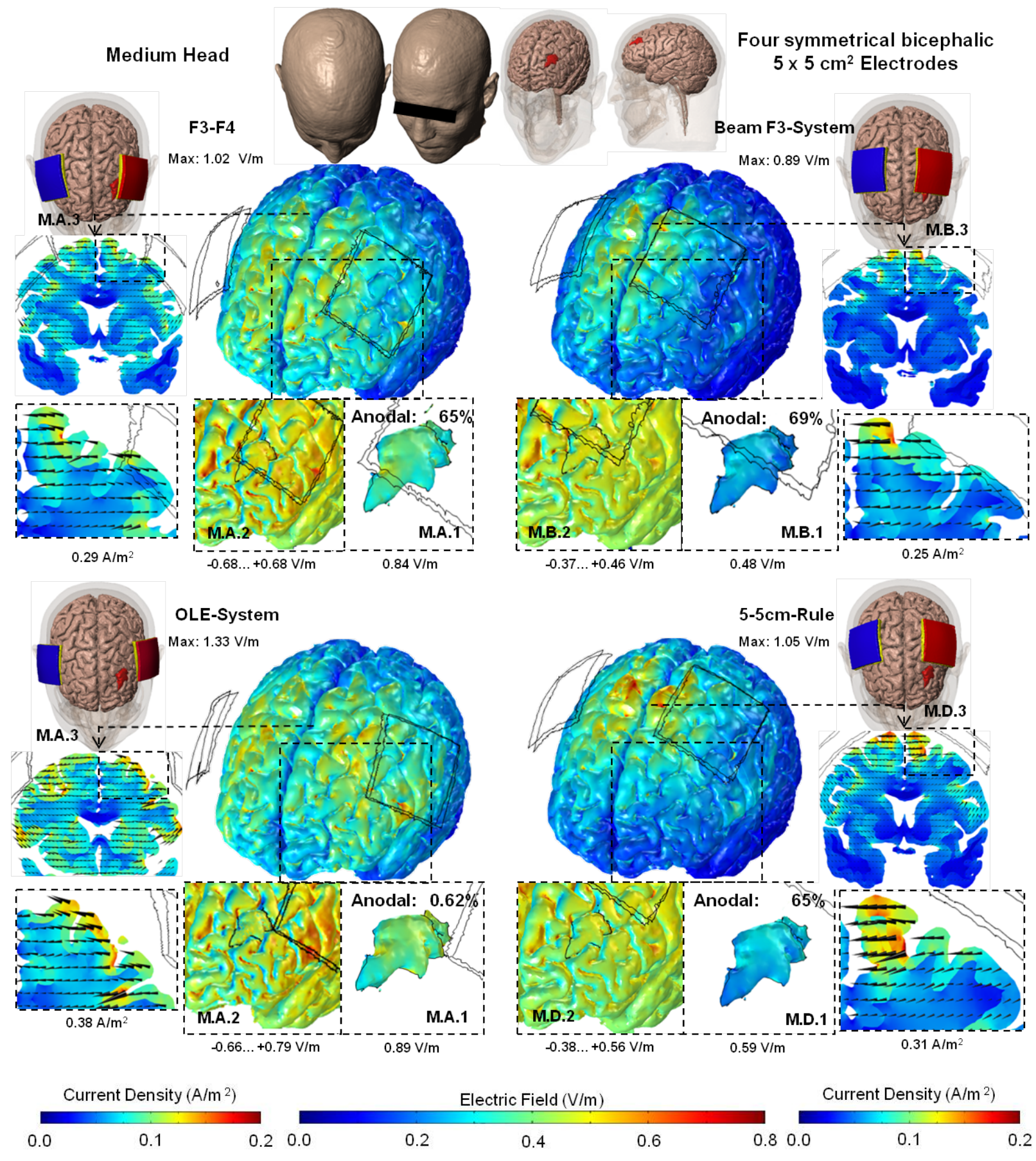

Figure 6: Comparison of brain current flow produced by four localization methods on a medium head size. Four methods are compared: "F3-F4" positioning according to the EEG 10-10 (M.B), "OLESystem" (M.C) and the "5-5cm-Rule" (M.D). 2mA, injected from the anode (red) to cathode (blue), resulted in the depicted electric field across the cortical surface. Cross-section views of the brain selected, for maximal intensity (M.A.3, M.B.3, M.C.3 and M.D.3), with magnified insets also showing the resulting vector field (cones), depict the produced current density distribution. The radial electric field across the lateral prefrontal region is shown with peak IDLPFC values for each case (M.A.2, M.B.2, M.C.2 and M.D.2); scaled to $\pm 0.8 \mathrm{~V} / \mathrm{m}$. The cortical electric field distribution in DLPFC (wireframe) is shown separately with the rate of anodal (inward) current flow (M.A.1, M.B.1, M.C.1 and M.D.1); scaled to $0.8 \mathrm{~V} / \mathrm{m}$. Resulting peak intensities are depicted adjacent to each plot. 

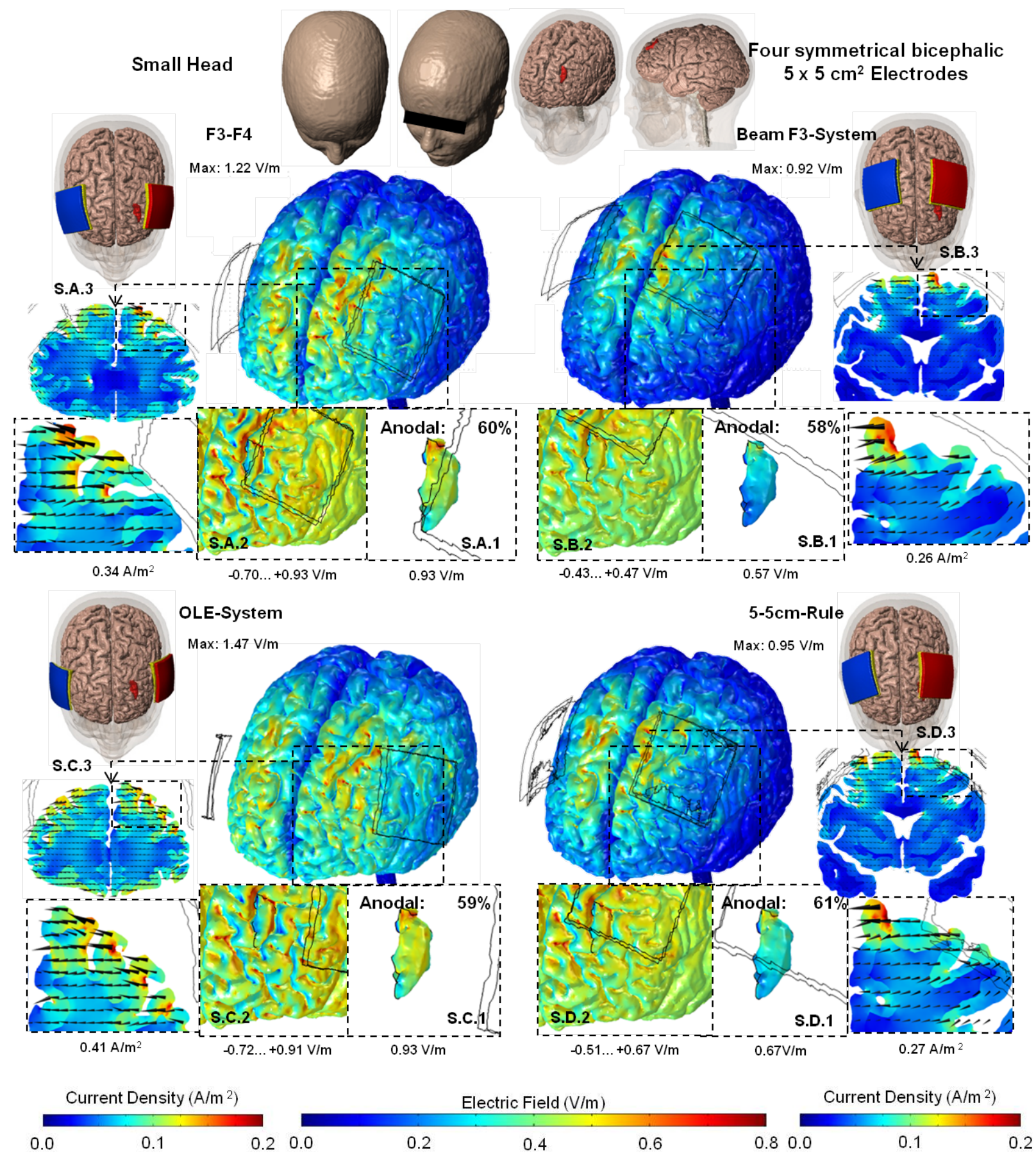

Figure 7: Comparison of brain current flow produced by four localization methods on a small head size. Four methods are compared: "F3-F4" positioning according to the EEG 10-10 (S.A), "Beam F3System" (S.B), "OLE-System" (S.C) and the "5-5cm-Rule" (S.D). 2mA, injected from the anode (red) to cathode (blue), resulted in the depicted electric field across the cortical surface. Cross-section views of the brain, selected for maximal intensity (S.A.3, S.B.3, S.C.3 and S.D.3), with magnified insets also showing the resulting vector field (cones), depict the produced current density distribution. The radial electric field across the lateral prefrontal region is shown with peak IDLPFC values for each case (S.A.2, S.B.2, S.C.2 and S.D.2); scaled to $\pm 0.8 \mathrm{~V} / \mathrm{m}$. The cortical electric field distribution in DLPFC (wireframe) is shown separately with the rate of anodal (inward) current flow (S.A.1, S.B.1, S.C.1 and S.D.1); scaled to $0.8 \mathrm{~V} / \mathrm{m}$. Resulting peak intensities are depicted adjacent to each plot. 


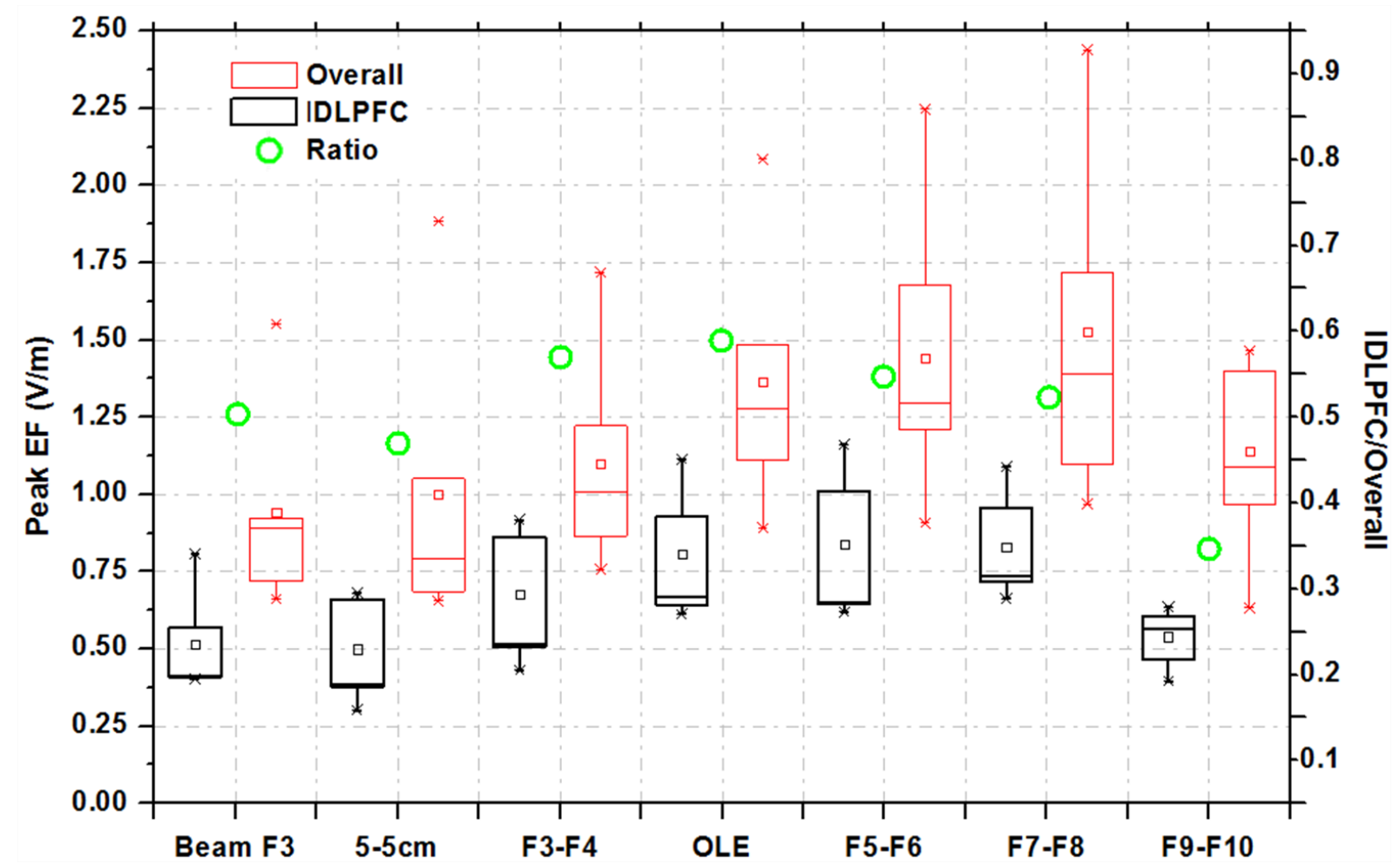

Figure 8: Peak electric field variability across six heads in IDLPFC (black) and overall cortex (red) illustrated aacross six subjects as box plot for each montage. The ratio of IDLPFC to overall cortical peak electric field is additionally depicted as circular rings (green). 

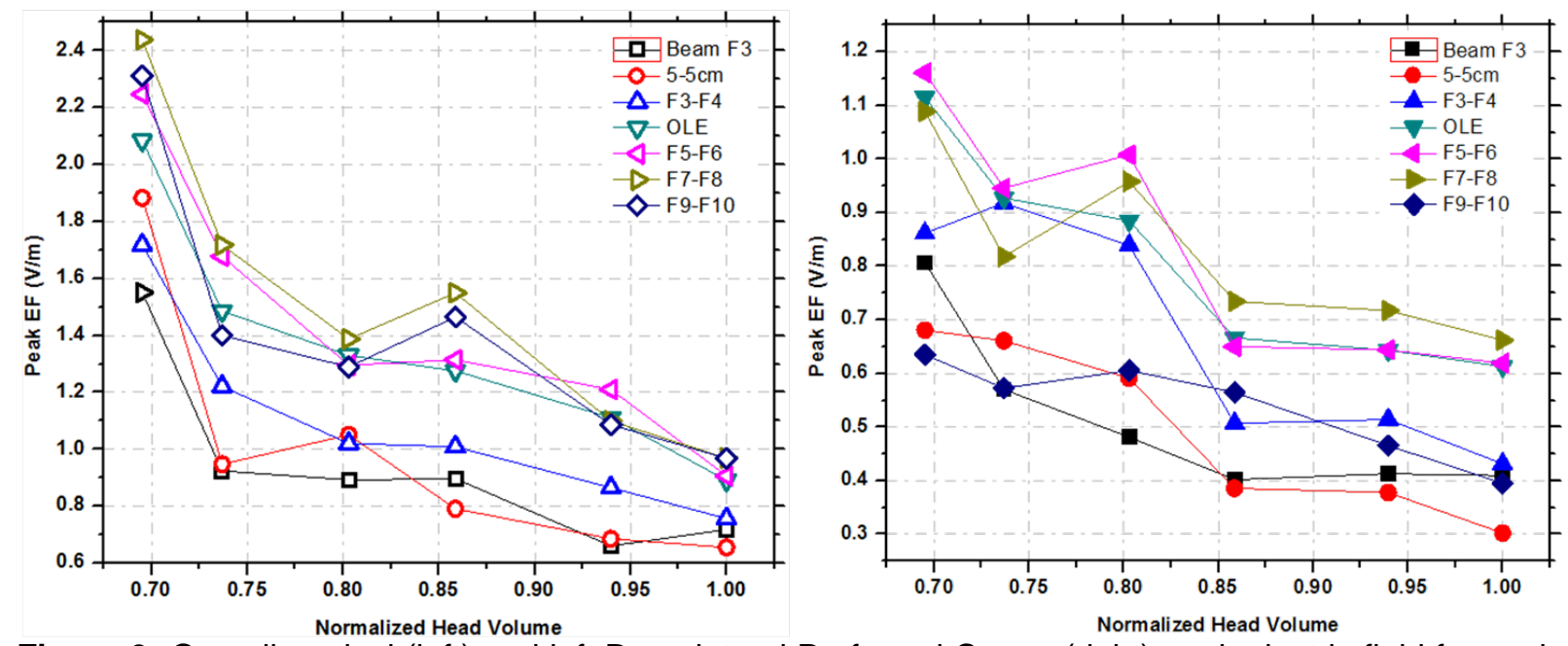

Figure 9: Overall cortical (left) and left Dorsolateral Prefrontal Cortex (right) peak electric field for each montage over normalized head volume. Montage-specific peak electric field depicted over each head tissue volume (measured between $\mathrm{C} 2$ (axis) and the vertex) relative to the MNI standard head. The extra small (XS), small (S), medium (M), large (L), extra large (XL) and the MNI standard head are shown from left to right. 

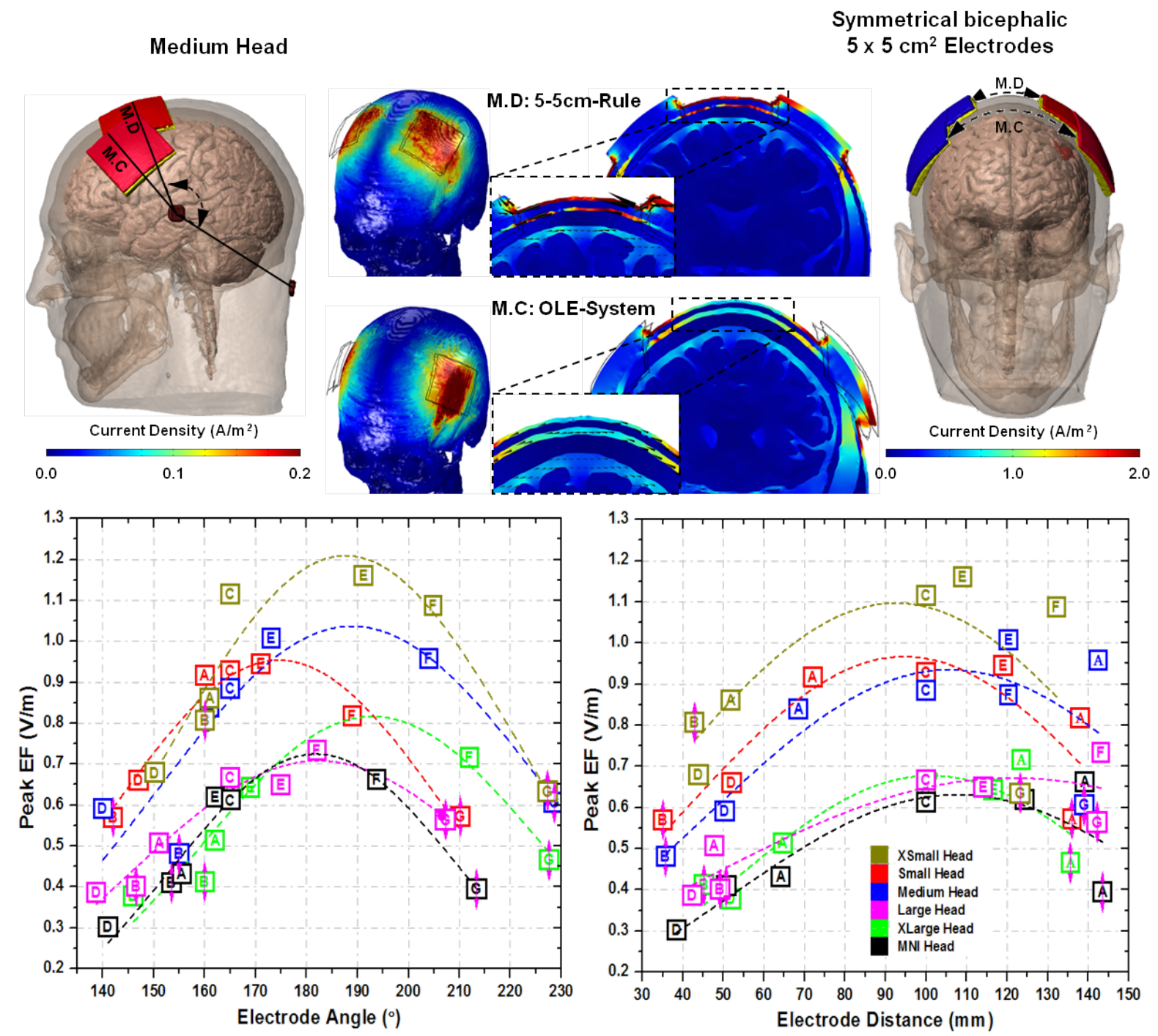

Figure 10: (Top) Exemplary illustration of volumetric current density distribution on the skull and coronal slice plot through all tissue compartments of a medium head. Current density distribution depicting current injection through the skull (left), scaled to $0.2 \mathrm{~A} / \mathrm{m}^{2}$, and a coronal slice plot through all tissue compartments; scaled to $2.0 \mathrm{~A} / \mathrm{m}^{2}$, illustrating scalp shunting (right) for electrode positioning according to the "OLE-System" (M.C) and " $5-5 \mathrm{~cm}$-Rule" (M.D). (Bottom) Peak electric field in IDLPFC depending on electrode positioning angle and distance, including a three-parameter exponential function trend line (dashed), depending on superior electrode edge distance on the scalp (right) and EasyStrap angle between occipital- and electrode-strap (left) for positioning according to F3-F4 (A), Beam F3-System (B), OLE-System (C), 5-5cm-Rule (D), F5-F6 (E), F7-F8 (F) and F9-F10 (G) for the investigated MNI (black), extra large (green), large (magenta), medium head (blue), small (red) and extra small head (dark green). 
Table 1: Tissue compartment volume for MNI standard (MNI), extra large (XL), large (L), medium (M), small (S) and extra small (XS) head sizes.

\begin{tabular}{c|c|c|c|c|c|c}
\hline \multicolumn{7}{c}{ Tissue Volume $\left(\mathrm{cm}^{3}\right)$} \\
\hline Tissue & MNI & XL & L & M & S & XS \\
\hline Skin & $1,029.1$ & 915.2 & 706.7 & 643.1 & 519.3 & 587.2 \\
Fat & 315.3 & 487.5 & 854.9 & 396.5 & 276.1 & 225.9 \\
Skull & 590.9 & 537.0 & 396.4 & 490.4 & 468.6 & 501.0 \\
CSF & 349.4 & 390.5 & 362.5 & 361.3 & 379.7 & 294.8 \\
Air & 60.7 & 85.4 & 35.1 & 31.7 & 48.8 & 21.4 \\
White-Matter & 639.5 & 516.3 & 421.3 & 508.2 & 523.2 & 467.3 \\
Gray-Matter & 925.1 & 740.7 & 580.9 & 708.1 & 666.1 & 620.0 \\
IDLPFC & 1.9 & 3.2 & 0.9 & 1.8 & 0.9 & 2.0 \\
\hline Total & $3,911.9$ & $3,675.8$ & $3,358.7$ & $3,141.1$ & $2,882.7$ & $2,719.6$
\end{tabular}


Table 2: Rate of anodal (inward) current flow in IDLPFC for each montages on an MNI standard (MNI), extra large (XL), large (L), medium (M), small $(S)$ and extra small $(X S)$ head.

\begin{tabular}{|c|c|c|c|c|c|c|}
\hline \multicolumn{7}{|c|}{$\begin{array}{c}\text { Rate of anodal current flow in IDLPFC (\%) } \\
\text { within radial EF range }(\mathrm{V} / \mathrm{m})\end{array}$} \\
\hline & $\mathrm{MNI}$ & $X \mathrm{~L}$ & $\mathrm{~L}$ & $M$ & $S$ & $X S$ \\
\hline Beam F3 & $\begin{array}{c}100 \\
-0.03 \ldots+0.35\end{array}$ & $\begin{array}{c}70 \\
-0.22 \ldots+0.39\end{array}$ & $\begin{array}{c}65 \\
-0.32 \ldots+0.40\end{array}$ & $\begin{array}{c}69 \\
-0.37 \ldots+0.46\end{array}$ & $\begin{array}{c}58 \\
-0.43 \ldots+0.47\end{array}$ & $\begin{array}{c}66 \\
-0.75 \ldots+0.73\end{array}$ \\
\hline $5-5 \mathrm{~cm}$ & $\begin{array}{c}100 \\
-0.02 \ldots+0.27\end{array}$ & $\begin{array}{c}64 \\
-0.24 \ldots+0.31\end{array}$ & $\begin{array}{c}58 \\
-0.32 \ldots+0.37\end{array}$ & $\begin{array}{c}65 \\
-0.38 \ldots+0.56\end{array}$ & $\begin{array}{c}61 \\
-0.51 \ldots+0.67\end{array}$ & $\begin{array}{c}63 \\
-0.64 \ldots+0.61\end{array}$ \\
\hline F3-F4 & $\begin{array}{c}100 \\
-0.03 \ldots+0.36\end{array}$ & $\begin{array}{c}69 \\
-0.23 \ldots+0.39\end{array}$ & $\begin{array}{c}62 \\
-0.40 \ldots+0.50\end{array}$ & $\begin{array}{c}65 \\
-0.68 \ldots+0.68\end{array}$ & $\begin{array}{c}60 \\
-0.70 \ldots+0.93\end{array}$ & $\begin{array}{c}65 \\
-0.78 \ldots+0.82\end{array}$ \\
\hline OLE & $\begin{array}{c}100 \\
-0.11 \ldots+0.54\end{array}$ & $\begin{array}{c}56 \\
-0.61 \ldots+0.52\end{array}$ & $\begin{array}{c}58 \\
-0.52 \ldots+0.64\end{array}$ & $\begin{array}{c}62 \\
-0.66 \ldots+0.79\end{array}$ & $\begin{array}{c}59 \\
-0.72 \ldots+0.91\end{array}$ & $\begin{array}{c}65 \\
-1.01 \ldots+0.94\end{array}$ \\
\hline F5-F6 & $\begin{array}{c}100 \\
-0.08 \ldots+0.52\end{array}$ & $\begin{array}{c}56 \\
-0.61 \ldots+0.52\end{array}$ & $\begin{array}{c}56 \\
-0.51 \ldots+0.63\end{array}$ & $\begin{array}{c}62 \\
-0.75 \ldots+0.75\end{array}$ & $\begin{array}{c}59 \\
-0.74 \ldots+0.94\end{array}$ & $\begin{array}{c}65 \\
-1.07 \ldots+0.95\end{array}$ \\
\hline F7-F8 & $\begin{array}{c}99 \\
-0.18 \ldots+0.54\end{array}$ & $\begin{array}{c}53 \\
-0.58 \ldots+0.64\end{array}$ & $\begin{array}{c}53 \\
-0.55 \ldots+0.62\end{array}$ & $\begin{array}{c}60 \\
-0.68 \ldots+0.70\end{array}$ & $\begin{array}{c}59 \\
-0.64 \ldots+0.80\end{array}$ & $\begin{array}{c}64 \\
-1.07 \ldots+0.89\end{array}$ \\
\hline F9-F10 & $\begin{array}{c}96 \\
-0.15 \ldots+0.37\end{array}$ & $\begin{array}{c}51 \\
-0.43 \ldots+0.41\end{array}$ & $\begin{array}{c}52 \\
-0.41 \ldots+0.43\end{array}$ & $\begin{array}{c}59 \\
-0.43 \ldots+0.40\end{array}$ & $\begin{array}{c}61 \\
-0.31 \ldots+0.48\end{array}$ & $\begin{array}{c}61 \\
-0.59 \ldots+0.53\end{array}$ \\
\hline
\end{tabular}

